\title{
Efeitos da Terapia por Contensáo Induzida em longo prazo em pacientes pós-AVC
}

\author{
Effects of long term Constraint-Induced Movement Therapy on patients after Stroke
}

Roberta Trevizan Gamba ${ }^{1}$, Daniel Marinho Cezar da Cruz ${ }^{2}$

\section{RESUMO}

Com o aumento da população idosa tem-se um expressivo crescimento das doenças crônicas, uma delas é o Acidente Vascular Cerebral. A lesão provoca, dentre suas várias sequelas, a hemiplegia, a qual dificulta a mobilização do membro superior e inferior de um hemicorpo, diminuindo a funcionalidade e a qualidade de vida do indivíduo acometido pelo Acidente Vascular Cerebral. A Terapia por Contensão Induzida é uma das diversas terapias, utilizadas para reduzir as incapacidades que prejudicam a funcionalidade do membro afetado. Esta revisão de literatura teve como objetivo identificar se a Terapia por Contensão Induzida, aplicada ao membro superior de indivíduos hemiplégicos, por Acidente Vascular Cerebral, manteve seus resultados em longo prazo. Foram analisados seis artigos que aplicaram este tipo de terapia em seus sujeitos e os observaram durante pelo menos um mês após o pós-teste. Como resultado, identificou-se que foram utilizados diferentes tipos de avaliaçôes, as quais calculavam o tempo de realizaçáo das atividades, quantidade e qualidade de uso dos membros superior comprometido. A revisão mostrou que a terapia por contensão induzida tende a manter seu resultado positivo, porém com algumas quedas dos escores das avaliaçóes utilizadas sem, no entanto, haver uma regressão da pontuação inicial dos sujeitos no nível do pré-teste.

Unitermos. Terapia por Contensão Induzida, Doenças Cerebrovasculares, Hemiplegia, Membro Superior, Terapia Ocupacional.

Citaçáo. Gamba RT, Cruz DMC. Efeitos da Terapia por Contensão Induzida em longo prazo em pacientes pós-AVC.
Trabalho realizado para conclusão do curso em pós-graduaçáo em Gerontologia pelo Instituto de Ensino e Pesquisa Albert Einstein-IEP, Sáo PauloSP, Brasil.

1. Terapeuta Ocupacional, pós-graduanda em Gerontologia pelo Instituto de Ensino e Pesquisa Albert Einstein, São Paulo-SP, Brasil.

2. Terapeuta Ocupacional, Doutorando em Educaçẩo Especial pela Universidade Federal de São Carlos-UFSCar, Professor Assistente do Departamento de Terapia Ocupacional da UFSCar, São Carlos-SP, Brasil.

\begin{abstract}
With the increasing of elderly population there is an expressive augmentation in chronic diseases, one of which is stroke. The injury causes, among its various disabilities, which hinders the mobilization of the upper and lower one hemisphere, reducing the functionality and quality of life of the person affected by stroke. The ConstraintInduced Movement Therapy is one of several kind of therapies used to reduce the disabilities that impair the functionality of the affected limb. This literature review of Constraint-Induced Movement Therapy, applied to the upper limb of hemiplegic individuals, for stroke, kept the long-term outcomes. Six articles that applied this therapy in its subjects were analyzed as well as observed for at least a month after the post-test. As a result, the authors identified that different types of assessments, were used which calculated the time to perform activities, the quantity and quality of use of affected upper Limb. The review showed that Constraint-Induced Movement Therapy tends to maintain its positive result, but with a few decreases in scores of the evaluations used without, however, be a regression of the initial score of the subjects in the pretest level.
\end{abstract}

Keywords. Constraint-Induced Movement Therapy, Stroke, Upper Extremity Paresis, Occupational Therapy.

Citation. Gamba RT, Cruz DMC. Effects of long term ConstraintInduced Movement Therapy on patients after Stroke. 


\section{INTRODUÇÃO}

É possível observar nos últimos anos uma redução no número de crianças e jovens e um aumento da população com idade igual ou superior a 60 anos e, consequentemente, passando por uma fase de transiçáo demográfica. Com o envelhecimento populacional, em 2025 espera-se um crescimento de $223 \%$ desta população. Segundo a OMS o Brasil será o sexto país do mundo em número de idosos ${ }^{1}$.

Este envelhecimento costuma vir acompanhado por um aumento da situação de dependência na população, o que implica em um aumento de custos públicos e que pode vir a sobrecarregar as famílias responsáveis por esses idosos ${ }^{2}$.

Com o crescimento da população idosa tem-se como consequência um aumento das doenças não transmissíveis tornando as doenças crônicas uma das maiores causas de morte e invalidez ${ }^{1}$. Isto cria um grande desafio para o governo que no período de 2000 a 2005 teve aproximadamente um aumento de $38 \%$ a $60 \%$ nos gastos com a população com 60 anos ou mais ${ }^{3}$.

A doença crônica não pode ser motivo para dependência, pois o idoso acometido por alguma moléstia deve ter uma vida saudável e com o máximo de autonomia, preservando suas capacidades funcionais, visto que a dependência é um fator de risco muito importante para mortalidade, mais que a própria doença limitante ${ }^{4}$.

Dentro das várias doenças crônicas que causam limitaçóes e que têm como um dos fatores de risco a idade avançada, temos o Acidente Vascular Cerebral (AVC). Uma de suas sequelas é a hemiparesia; comprometimento do membro superior e inferior de um mesmo lado do corpo, caracterizada por um padrão rígido nos músculos flexores do membro superior e nos músculos extensores do membro inferior ${ }^{5}$. O AVC é a segunda causa de morte no mundo e metade das pessoas que sobrevivem têm um grande grau de dependência, principalmente na realização da suas Atividades de Vida Diária (AVDs) ${ }^{6}$. Segundo a OMS, o Brasil é o sexto na lista dos países com maior número de vítimas de $\mathrm{AVC}^{7}$. Seis meses após o percurso do AVC 65\% das pessoas não conseguem incorporar o lado afetado em suas AVDs, apenas 25\% conseguem retornar as suas atividades sociais e a qualidade de vida tende a ser maior naqueles indivíduos que melhor recupera- ram suas funcionalidades. Esse quadro pode ser revertido consideravelmente através de terapias de reabilitação ${ }^{8}$.

Hoje existem várias terapias de reabilitação com êxito comprovado5. E uma das terapias que têm demonstrando grandes resultados é a Terapia por Contensão Induzida (TCI $)^{9,10}$, que tem como foco principal a desprogramação do desuso motor e não apenas da disfunção motora, manifestada pelo indivíduo hemiparético. Nesta terapia, o paciente tem seu membro não afetado imobilizado durante $90 \%$ do tempo em que permanecer acordado, recebe sessóes de terapias para treino do uso do membro superior parético e é estimulado a reproduzir os movimentos nas suas AVDs. Esta terapia baseia-se no fundamento de que o uso forçado do membro superior parético irá favorecer o aprendizado motor ${ }^{11}$. Desta forma, são características dessa técnica de tratamento, o uso máximo do membro afetado, a inserção do sujeito em atividades funcionais e cotidianas e a restrição do membro superior saudável nas tarefas do dia a dia.

Estudo recente evidencia que a TCI é um tratamento potente para melhorar a movimentação funcional do membro hemiplégico, do indivíduo com sequelas de $\mathrm{AVC}^{12}$. Outro estudo, realizado antes do tratamento com TCI e após o tratamento, através da imagem por ressonância magnética, revelou mudanças nas estruturas cerebrais, na região cinzenta das áreas sensórias e motoras do cérebro, acompanhadas pela melhora da função motora espontânea do membro com hemiparesia. Foram também identificadas mudanças no hipocampo que é uma estrutura que se relaciona com o processo de aprendizagem e memória ${ }^{13}$.

Este artigo de revisão tem por propósito apresentar o efeito dos resultados da TCI em sujeitos pós- AVC ao longo do tempo.

\section{MÉTODO}

\section{Critérios de seleçáo}

Os artigos foram selecionados por meio de três critérios de inclusão: a) publicação nos últimos cinco anos (2005-2009); b) pesquisas experimentais com pré e pósteste; c) que acompanharam os sujeitos depois do pósteste. Foram quatro os critérios de exclusão: a) pesquisas cuja amostra foi constituída de crianças; b) pesquisas cujo objeto de estudo não foi a reabilitaçấo de membros su- 
periores; c) pesquisas que utilizaram um protocolo modificado de TCI; d) pesquisas que não exemplificavam a classificação do AVC em crônico, sub-agudo e agudo.

\section{Fontes da coleta de dados}

Todos os artigos do estudo foram recuperados na base de dados PubMed, por ser uma base representativa de artigos da área da saúde, sendo utilizado como palavras-chave para a busca combinada (and ou or): - stroke;Constraint-Induced Movement; -long term; -folow-up. Além dos critérios supracitados, após a recuperação de nove resumos, foram considerados apenas aqueles cuja pesquisa era com seres humanos e com sujeitos de idade limite de 45 anos.

Após estes critérios estabelecidos, foram selecionados seis artigos, dos nove encontrados, sendo que dois artigos não foram possíveis de serem analisados na íntegra e seus resumos não classificavam o tipo de AVC que os pacientes do estudo apresentavam no momento da pesquisa.

\section{Tratamento e análise dos dados}

Para tratamento e análise dos dados, a partir da análise qualitativa em categorias, por conteúdo, optou-se por separar os artigos em dois grupos distintos:

1) Grupo Agudo e subagudo (GA): neste grupo, os resultados foram encontrados em pacientes que receberam a terapia com menos de um ano após o primeiro AVC.

2) Grupo crônico (GC): Neste grupo os resultados foram encontrados em pacientes que receberam a terapia após um ano de seu primeiro AVC.

\section{RESULTADOS}

Dada a importância da avaliação para a prática de reabilitação e para as pesquisas, cabe destacar brevemente as avaliações utilizadas nas pesquisas revisadas, a fim de melhor compreensão do que estas se propóem a medir.

Em relação às avaliaçóes utilizadas como critérios para mensuração dos resultados das pesquisas, observouse a predominância de algumas avaliaçóes. Da amostra de seis (06) artigos recuperados, foram utilizadas as seguintes avaliaçóes:

a) Motor Activity $\log (\mathrm{MAL})^{14}$ uma entrevista semi-estruturada que avalia a opinião do sujeito em 30 atividades e é subdividida em duas escalas:

- AOU que é a quantidade de uso do MS e;

- QOM que avalia a qualidade do movimento do MS;

b) WolfMotor Function Test (WMFT) ${ }^{14}$ que consiste na realização de 15 tarefas cronometradas e mais duas tarefas de força;

c) Fugl-Meyer Assesment (FMA) ${ }^{15}$, que avalia medidas de habilidade motora, coordenação, velocidade, amplitude de movimento, sensibilidade e dor da extremidade superior;

d) Stroke Impact Scale (SIS) $)^{14}$ que é uma escala que avalia a incapacidade do paciente com AVC. Tem critérios como força, pensamento/memória, AVDs/Atividade de Vida Prática (AVP), participação social e domínio físico.

\section{Grupo Crônico (GC)}

Quatro dos seis artigos foram realizados com pacientes com AVC crônico, sendo que entre eles existe um estudo de caso com uma paciente que recebeu sessóes de TCI e os outros dois foram pesquisas que selecionaram aleatoriamente indivíduos que se enquadravam no protocolo da terapia por contensão induzida. Em ambos os estudos, os pacientes eram avaliados antes e depois por terapeutas "cegos" em relação à pesquisa.

No estudo de caso (Estudo 1 da Tabela 1) foi utilizada como avaliação no pós e pré-testes: MAL e WMFT ${ }^{16}$. Neste estudo o paciente teve melhora na WMFT com uma média de -0.84 segundos entre pré e o pós-teste, indicando uma diminuição do tempo na realização das atividades, esta média após 4 anos, foi de -1.14 segundos, e -0.99 segundos, após 5 anos. A MAL AOU no pré-teste era de 1.18, no pós-teste subiu para 3.73, e após 4 e 5 anos teve uma queda, mas mantendo-se acima da pontuação do pré-teste. A MAL e QOM manteve uma curva semelhante da AOU.

No estudo $2^{17}$ foram escolhidos aleatoriamente 20 pacientes e foi utilizado para as avaliaçóes: FMA, Graded Wolf/Motor Function Test (GWMFT), uma versão adaptada da WMFT, para avaliar comprometimento motor moderado a severo, e Motor Activity Log (MAL). Dos 20 sujeitos que participaram da terapia, 17 compareceram nas avaliaçóes, que ocorreram após um mês do tratamento. O FMA teve uma variação média de 5,6 pontos, 
Tabela 1

Diferença média dos resultados das avaliaçōes

\begin{tabular}{|c|c|c|c|c|c|c|c|}
\hline \multicolumn{2}{|c|}{ Medidas/Avaliaçáo } & \multicolumn{4}{|c|}{ GC } & \multicolumn{2}{|c|}{ GA } \\
\hline & & ${ }^{*}$ Estudo1 & Estudo2 & Estudo3 & ${ }^{* *}$ Estudo4 & Estudo5 & ${ }^{* * *}$ Estudo6 \\
\hline \multirow{3}{*}{$\begin{array}{c}\text { MAL } \\
\text { (AOU) }\end{array}$} & Pós-pré & 1.55 & I & NA & NA & 1.73 & 1.16 \\
\hline & Long-pós & $-1.53 /-1.43$ & I & NA & NA & 0.89 & $-0.13 / 0.04$ \\
\hline & Long-pré & $1.02 / 1.12$ & 1 & 3.2 & $1.71 / 1-6$ & 2.62 & $1.03 / 1.20$ \\
\hline \multirow{3}{*}{$\begin{array}{c}\text { MAL } \\
\text { (QOM) }\end{array}$} & Pós-pré & 2.23 & I & 2.6 & 1.77 & 1.6 & 1.04 \\
\hline & Long-pós & $1.53 / 1.53$ & I & -2.6 & $-0.05 / 0.03$ & 1.2 & $-0,02 / O .12$ \\
\hline & Long-pré & $1.53 / 1.53$ & 1 & 2.9 & $1.72 / 1.80$ & 2.8 & $1.02 / 1.16$ \\
\hline \multirow{3}{*}{$\begin{array}{c}\text { WMFT } \\
\text { (segundos) }\end{array}$} & Pós-pré & -0.87 & -2 & -63 & 0.20 & I & -0.57 \\
\hline & Long-pós & $-0.27 /-0,12$ & -0.2 & -15 & $0.51 /-2.39$ & I & $-0.07 /-0.15$ \\
\hline & Long-pré & $-1.14 /-0.99$ & -2.02 & -48 & $0.71 /-2.19$ & I & $-0.64 /-0.72$ \\
\hline \multirow[t]{3}{*}{ FMA } & Pós-pré & I & 1 & 6 & 6 & 18.45 & 1 \\
\hline & Long-pós & I & I & 0 & $6 / 1$ & 5.55 & I \\
\hline & Long-pré & I & I & 6 & $12 / 7$ & 24 & I \\
\hline
\end{tabular}

Long = avaliação após pós-teste; $\mathrm{NA}$ = nẫo teve dados suficientes para o cálculo; / = a avaliação não foi usada no estudo;

* 4/5 anos após pós-teste; ${ }^{* *} 1 / 6$ meses após pós-teste; ${ }^{* * *} 1 / 1$ anos após pós-teste

entre pré e pós-tratamento, não tendo diferença um mês após o tratamento. O GWMFT variou em média de 0,5 entre o pré e o pós-teste, e não teve alteraçôes significantes após um mês do tratamento. A MAL AOU teve uma variação média de 1,7 entre o pré-tratamento e após um mês de tratamento, e o QOM teve uma variação média de 1,5 entre o pré e o pós-teste, sem ter variação depois de um mês.

No estudo $3^{15}$ foram selecionados 27 sujeitos (todos receberam a terapia), mas dois não passaram pela avaliação. Foi aplicada a WMFT, no pós e pré-teste para a avaliação. Na WMFT, teve-se variação de -2.3 segundos no tempo da realização das atividades entre o pré e pós-teste. Após três meses do pós-teste a variação média no tempo da realização das atividades, comparado com o pré-teste foi de $-2,2$ segundos.

No estudo $4^{18}$ a TCI foi aplicada em 7 sujeitos e sua eficácia foi verificada 1 mês e 6 meses após o tratamento. A diferença de resultado entre o pós e pré-teste da WMFT foi de 0.2 segundos, porém o pré-tratamento teve como pontuação 11.19 segundos e, seis meses depois esta pontuação foi de 9.00 segundos. A MAL AOU teve um aumento na pontuação média, quando comparado o resultado do pré-teste, com os resultados um mês após o tratamento e 6 meses após o tratamento, sendo estas pontuaçóes respectivamente $0.63,2.34$ e 2.28 (não foi possível fazer a média do pós-teste). $\mathrm{O}$ mesmo ocorreu com a MAL QOM (pré-teste 0-67, pós-teste 2.44, 1 mês depois do tratamento 2.39 , seis meses após o tratamento 2.39). A FMA do membro superior teve uma diferença média entre o pós e o pré-teste de 6 pontos. Quando esta diferença foi feita com pós-teste e um mês e seis meses após tratamento foi respectivamente 12 e 7 pontos.

\section{Grupo Agudo e Subagudo (GA)}

Dos seis artigos usados nesta revisão, dois eram com indivíduos que sofreram AVC há menos de 12 meses. Três artigos envolveram pesquisas cujos participantes foram escolhidos aleatoriamente e que se enquadravam no protocolo de TCI e as avaliaçóes foram aplicadas por terapeutas "cegos" em relação à pesquisa.

O estudo $5^{19}$ avaliou e comparou os resultados das avaliaçóes da TCI com os resultados da terapia de reabilitação normal pós-AVC em 16 pacientes. Estes são comparados após o tratamento e depois de 02 meses da finalização do mesmo. Neste artigo foi utilizado para avaliação FM, MAL, e a estimulação magnética transcraniana (EMT). A FM apresentou uma variação média de 18.45 entre o pós e pré-teste, e entre o pré-teste e dois meses após a intervenção, a diferença foi de 5.55 pontos. 
A variação média da MAL entre o pós e pré-teste foi de 1.73 , e entre o pré-teste e dois meses após a intervenção, foi de 1.20. Na GPT, quando realizadas as comparaçóes anteriores também foi possível identificar melhoras. Após a TCI e após 3 meses do tratamento foi possível observar um alargamento da zona de estabilidade cortical.

O estudo $6^{14}$ em que 105 sujeitos participaram do pré-teste, 98 do pós-teste, 80 da avaliação, após um ano, e 68, da avaliação pós dois anos, foi usada como avaliação WMFT, MAL e SIS. O WMFT apresentou uma variação média de -10.13 segundos entre o pré e pós-teste, já entre o pós e um ano e dois anos após o tratamento, a variação foi respectivamente, 0.91 segundos e 0.31 segundos, porém o tempo médio para realização das atividades era inicialmente 22.72 segundos e depois de dois anos esse tempo passou para 13.88 segundos, tendo então, uma diferença média de -8.84. A pontuação da MAL AOU referiu uma diferença média do pós para o pré-teste de 1.16 e a pontuação média do pós-teste, quando comparado com a pontuação do primeiro e segundo ano após o tratamento, não teve grande diferença (pós-teste 2.53, um ano 2.40 e dois anos 2.57). A diferença média entre após dois anos de tratamento com pré-teste foi de 1.20. A SIS apresentou aumento na pontuação nos critérios: força, memória/ pensamento, AVD/AVP, participação social e controle físico quando comparado o pré-teste com a pontuação de dois anos após o tratamento.

\section{DISCUSSÃO}

$\mathrm{O}$ estudo de caso foi realizado com uma paciente de $57 \operatorname{anos}^{16}$. Os artigos randomizados ${ }^{15,17,18}$ do GC tinham média de idade no intervalo de [53] a [57,5] e todos continham amostra $(\mathrm{N})$ masculina maior que a feminina, o que pode ser justificado pelo fato do sexo masculino, até os 80 anos de idade, ser um dos fatores de risco para doenças vasculares cerebrais ${ }^{20}$. Os artigos apontaram que não é significante a diferença de pontuação das avaliações, quando é levado em consideração a idade ou o sexo.

Os artigos do GC mostraram que o tempo na realização nas atividades é diminuído após a terapia por contensão induzida, e que este tempo, apesar de aumentar após um intervalo de tempo do tratamento, se mantêm menor do que o tempo gasto antes da terapia (pós-teste). Como a função e a qualidade do movimento também seguem resultados semelhantes, porém em alguns casos houve melhora dos escores de qualidade de movimento após um intervalo de tempo decorrente do tratamento, o que é uma evidência de que o paciente continua a realizar os movimentos após o tratamento e assim a melhora na qualidade do uso.

No estudo de caso que reaplicou as avaliaçóes em um maior espaço de tempo (cinco anos), foi possível constar que o tempo para realização das atividades aumentou após os cinco anos, e a quantidade e qualidade de movimento teve uma queda no escore, porém manteve-se maior que o escore da avaliação inicial (pré-teste).

Dos artigos do GA, apenas um colocou a média de idade dos participantes, que foi de 63,1 anos ${ }^{19}$. Neste grupo também foi maior o número de sujeitos homens, como já era esperado devido ao fator de risco ${ }^{20}$.

Nos artigos do GA foi observada uma melhora na função motora, que continuou após algum tempo depois do tratamento até mesmo tendo melhora na pontuação (em alguns casos). A quantidade e qualidade do movimento também seguiu a mesma tendência. $O$ estudo 6 utilizou uma quantidade grande de sujeitos com um acompanhamento de até dois anos ${ }^{14}$, tendo assim uma melhor relevância e demonstrou bons resultados da TCI após o tratamento.

Apesar do número pequeno de artigos quando comparamos os resultados do GC com o do GA, é possível observar que os sujeitos do GA conseguem melhorar os escores depois de um intervalo de tempo após o tratamento, evento que não ocorreu com GC, isto indica a importância do tratamento ocorrer o mais precoce possível, a fim de obter melhores resultados e assim um aumento da qualidade de vida.

Os artigos também evidenciam que, talvez, seja necessária uma manutenção da TCI, pois mesmo os pacientes não retornando à pontuaçáo do pré-teste obtiveram um declínio desta pontuação, que em hipótese, poderia ser melhorada com a manutenção do tratamento.

\section{CONCLUSÃO}

A despeito do número pequeno de artigos foi possível concluir que existe uma persistência dos resultados positivos da TCI em longo prazo, e que este resultado é mais satisfatório quando a reabilitação ocorre na fase 
aguda ou subaguda do AVC, podendo este tipo de terapia ser uma opção para reabilitação do indivíduo com AVC. São necessários mais estudos que acompanhem em longo prazo os pacientes que participarem de TCI, com amostras amplas e a realização de revisôes sistemáticas sobre as evidências para resultados mais confiáveis.

\section{REFERÊNCIAS}

1.Gontijo S. Envelhecimento Ativo: uma política de saúde. Brasília: Organizaçáo Pan Americana de Saúde, 2005, p2-62.

2.Camarano AA. Envelhecimento da população brasileira: contribuiçăo demográfica. In: Freitas EV, Py L, Cançado FAX, Doll J, Gorzoni ML. Tratado de Geriatria e Gerontologia. 2a . ed. Rio de Janeiro: Guanabara Koogan, 2006, p.87105.

3.Chaimowiez F. Epidemiologia e o envelhecimento no Brasil. In: Freitas EV, Py L, Cançado FAX, Doll J, Gorzoni ML. Tratado de Geriatria e Gerontologia. $2^{\text {a }}$. ed. Rio de Janeiro: Guanabara Koogan, 2006, p.106-30.

4.Veras R. Envelhecimento populacional contemporâneo: demandas, desafios e inovações. Rev Saúde Publica 2009; 43:548-54. http://dx.doi.org/10.1590/S0034-89102009005000025

5.Pereira LSM, Dias RC, Dias JMD, Gomes GC, Sitta MI. Fisioterapia em Gerontologia. In: Freitas EV, Py L, Cançado FAX, Doll J, Gorzoni ML. Tratado de Geriatria e Gerontologia. 2a . ed. Rio de Janeiro: Guanabara Koogan, 2006, p.1198-209.

6.Legg L, Drummond A, Langhorne P. Occupational therapy for patients with problems in personal activities of daily living after stroke: systematic review of randomized trials. BMJ;2007:335:894-5.

http://dx.doi.org/10.1136/bmj.39343.466863.55

7.Esther MJ, Steultjens MA, Dekker J, Bouter LM, Van de Nes JCM, Cup EHC, et al. Occupational Therapy for stroke patients: A Systematic Review. Stroke 2003;34;676-87.

http://dx.doi.org/10.1161/01.STR.0000057576.77308.30

8.Dobkin BH. Rehabilitation after stroke. N Engl J Med 2007; 352:1677-84. http://dx.doi.org/10.1056/NEJMcp043511

9.Miltner WHR, Bauder H, Sommer M, Dettmers C, Taub E. Effects of Cons-
traint-Induced Movement Therapy on patients with chronic motor deficits after stroke: a replication. Stroke 1999;30:586-92.

http://dx.doi.org/10.1161/01.STR.30.3.586

10.Page JS, Levine P, Leonard A, Szaflarski JP, Kissela BM. Modified Constraint-Induced Therapy in Chronic Stroke: Results of a Single Blinded Randomized Controlled Trial. Physical Therapy 2008;88:333-40.

http://dx.doi.org/10.2522/ptj.20060029

11.Sousa $\mathrm{RD}$. Terapia de restriçăo de membro superior não parético e induçâo do movimento em pacientes hemiparéticos. [dissertação]. Campinas: Faculdade de Ciências Médicas da Universidade Estadual de Campinas; 2008, 96p. 12.Miltimer WHR, Bauder H, Sommer M, Dettmers C, Taub E. Effects of modified constraint-induced therapy on patients with chronic motor deficits after stroke. Stroke. 1999:30:586-92.

http://dx.doi.org/10.1161/01.STR.30.3.586

13.Gauthier LV, Taub E, Perkins V, Ortmann M, Mark VW, Uswatte G. Remodeling the brain plastic structural brain changes produced by different motor therapies after stroke. Stroke 2008;39:1520-5.

http://dx.doi.org/10.1161/STROKEAHA.107.502229

14. Wolf SL, Winstein CJ, Miller JP, Thompson PA, Taub E, Uswatte G, et al. The EXCITE trial: retention of improved upper extremity function among stroke survivors receiving CI movement therapy. Lancet Neurol 2008;7:33-40. http://dx.doi.org/10.1016/S1474-4422(07)70294-6

15.Bonifer NM, Anderson KM, Arciniegas DB. Constraint-Induced Movement Therapy after stroke: efficacy for patients with minimal upper-extremity motor ability. Arch Phys Med Rehabil 2005;86:1867-73.

http://dx.doi.org/10.1016/j.apmr.2005.04.002

16.Rowe VT, Steven S, Wolf SL. Long term follow-up after Constraint-Induced Therapy: a case report of a chronic stroke survivor. Am Jour Occup Ther 2009;63:317-22.

17.Tarkka IM, Pitkanen K, Sivenius J. Paretic hand rehabilitation with Constraint-Induced Therapy after stroke. Am J Phys Med Rehabil 2005;84:501-5. http://dx.doi.org/10.1097/01.phm.0000166881.71097.9d

18. Bonifer NM, Anderson KM, Arciniegas DB. Constraint-induced therapy for moderate chronic upper extremity impairment after stroke. Brain Inj 2005;19:323-30.

http://dx.doi.org/10.1080/02699050400004302

19. Boake C, Noser EA, Ro T, Baraniuk S, Gaber M, Johnson R, et al. Constraint-Induced Therapy during early stroke rehabilitation. The Am Soc Neurorehab 2007;21:14-24.

20.Py MO. Doenças Cerebrovasculares. In: Freitas EV, Py L, Cançado FAX, Doll J, Gorzoni ML. Tratado de Geriatria e Gerontologia. 2a ${ }^{\text {a }}$ ed. Rio de Janeiro: Guanabara Koogan, 2006, p.333-47. 